BBA 75250

\title{
LOW-ANGLE X-RAY DIFFRACTION PATTERNS FROM A VARIETY OF MYELINATED NERVES
}

\author{
A. E. BLAUROCK AND C. R. WORTHINGTON
}

Department of Physics and Biophysics Research Division, Unvversity of Michigan, Ann Arbor, Mich. 48 I04 (U.S.A.)

(Recelved November 4 th, 1968)

SUMMARY

Low-angle X-ray diffraction patterns from a variety of myelinated nerves are described. Different kinds of patterns are obtained from peripheral nerve and central nervous system myelin. The intensity variation between the first five diffraction orders is the same for each variety of peripheral nerve myelin, and similarly, an intensity variation exists which is the same for each variety of central nervous system myelin. However, the intensity variation for myelinated nerves from fish is different from peripheral nerve and central nervous system myelin. New diffraction orders higher than five for both peripheral nerve and central nervous system myelin have been recorded.

\section{INTRODUCTION}

Specimens of live myelinated nerve when examined by low-angle $\mathrm{X}$-ray diffraction show a radial repeating unit of $150-185 \AA$, depending on the type of nerve. There are two main types of nerve which give different low-angle $\mathrm{X}$-ray diffraction patterns; peripheral nerve myelin (e.g. sciatic nerves and spinal roots) and central nervous system myelin (e.g. optic nerve, spinal cord and white brain material). The peripheral nerve myelin pattern ${ }^{1,2}$ consists of five orders $(h=I-5)$ of a radial repeating unit of $d \approx \mathrm{I}_{70-\mathrm{I} 85} \AA$. The central nervous system myelin pattern ${ }^{3,4}$ consists of only two orders of $d \approx \mathrm{I} 50-\mathrm{I} 6 \mathrm{o} \AA$.

In a previous theoretical study ${ }^{5}$ we have found the correction factors which must be applied to the X-ray intensities obtained from the low-angle pattern of nerve in order to proceed with any structural analysis, and naturally we were interested in examining the consequences of using corrected intensities in a structural analysis of nerve. We therefore set out to record low-angle patterns from a variety of myelinated nerves, to measure quantitatively the intensities from certain patterns and to search for any new aspects in the patterns not previously recorded by others. Due largely to the fact that our low-angle $\mathrm{X}$-ray camera ${ }^{6}$ is an improvement over the usual slit camera for obtaining low-angle $\mathrm{X}$-ray diffraction patterns of live nerve, we have recorded additional orders of diffraction out to a minimum spacing of I $^{-}-\mathrm{I} 6 \AA$. In this paper we describe some of our low-angle $X$-ray diffraction patterns obtained from a variety of nerves. The interpretation of our low-angle $\mathrm{X}$-ray patterns is treated in 
a later publication, although a letter describing an electron-density model for nerve has already appeared?.

\section{EXPERIMENTAL}

Low-angle X-ray diffraction patterns were obtained from the following nerves: frog (Rana pipiens) sciatic nerve, optic nerve and spinal cord; rat sciatic nerve and optıc nerve; chicken sciatic nerve and optic nerve; beef brain white matter; fish (Tilapıa mossambica) lateral line, optic nerve and spinal cord.

Freshly dissected nerves were immersed in frog Ringer's or Krebs-Ringer's solutions (Biochemist's Handbook, I96r) at room temperatures. Each nerve specimen together with excess solution was sealed in a thin-walled (Io $\mu$ thick) glass capillary tube.

The low-angle X-ray camera was the Jarrell-Ash version of an optically focusing X-ray diffraction camera ${ }^{6}$. The $\mathrm{X}$-ray source was a Jarrell-Ash microfocus tube. Most patterns were taken using only the first mirror (vertical) and a line focus (vertical) on the copper anode as used in a previous study ${ }^{8}$. Although this arrangement had resolution in one direction only, it had the advantage that the camera speed was increased over the normal operation. The first mirror of the $\mathrm{X}$-ray camera was as close to the $\mathrm{X}$-ray source as could be arranged ${ }^{9}$. This gave an additional increase in camera speed.

$\mathrm{X}$-ray patterns were normally taken with nickel-filtered copper $\mathrm{K} \alpha$ radiation except for a few patterns when the nickel filter was not used in order to increase camera speed. The specimen-to-film distance was normally set at $5.5 \mathrm{~cm}$, although it could be increased to $13.2 \mathrm{~cm}$ when required. Exposure times usually did not exceed I $2 \mathrm{~h}$ in order that the nerve myelin low-angle pattern did not change during exposure, although on occasions longer exposure times were used.

Preliminary experiments showed that fresh nerve specimens, sealed in glass capillaries with excess solution, did not change in any way over a period of at least I2 $\mathrm{h}$. In these experiments the time between dissection and the start of the X-ray exposure was less than $\mathrm{I} h$. Thus it can be argued that our wet nerve specimens had the same structure as live nerve. The peripheral nerve myelin patterns showed no changes for several days, whereas the central nervous system myelin patterns showed some line broadening and a small spacing increase after $24 \mathrm{~h}$.

$\mathrm{X}$-ray films were measured using a low-power microscope. The error in spacing measurement is estimated to be $\mathbf{I} \%$ for the shorter specimen-to-film distance. When the maximum specimen-to-film setting was used, the spacing measurement error was reduced to $0.5 \%$. However, the shorter specimen-to-film setting was normally used, and all radial repeat distances quoted in this paper are estimated to be accurate to I \% unless stated otherwise.

$\mathrm{X}$-ray patterns were recorded on Ilford Industrial G film. Either two or three films were placed in the cassette together in order to give an adequate range of intensities. An intensity range of about 300: I was covered. Densitometer tracings of the X-ray patterns were obtained on a Joyce-Loebl microdensitometer model MK III C. The background-scattering curve was subtracted from the densitometer tracing in the usual way. The integrated intensities, $I(h)$ where $h$ is the diffraction order, were measured either by integrating the areas under the diffraction peaks or 
by measuring the peak heights. Since the line widths of the first five orders of diffraction were identical, peak heights were used as a measure of intensity. The validity of using peak heights was established by comparing both methods of measuring intensity from several low-angle $\mathrm{X}$-ray patterns.

\section{Low-angle $X$-ray diffraction patterns}

Short exposures of $I \mathrm{~h}$ duration confirmed earlier observations ${ }^{\mathbf{2}, 4}$ that the peripheral nerve myelin patterns showed five orders of diffraction and the central nervous system myelin patterns showed two orders of diffraction. Fig. I shows a line focus low-angle X-ray diffraction pattern from frog sciatic nerve in Ringer's solution. The pattern was taken using copper $\mathrm{K} \alpha$ nickel-filtered radiation, a specimen-to-film distance of $5.5 \mathrm{~cm}$ and an exposure time of $\mathrm{I}$ h. The first five diffraction orders $(h=$ I-5) of a radial repeat distance $d=$ I7I $\AA$ are visible. Previous peripheral nerve myelin low-angle patterns ${ }^{2,4}$ show the same five diffraction orders as in Fig. r. However, the line focus pattern in Fig. I shows very sharp diffraction lines; previous
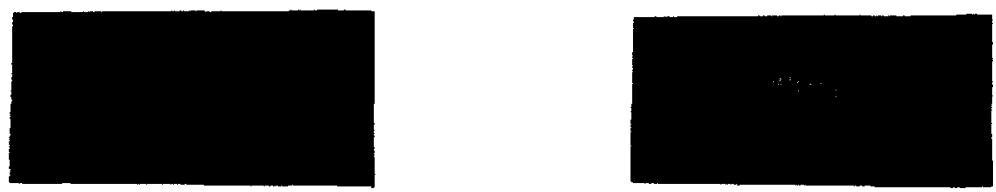

Fig. I. A line-focus low-angle X-ray diffraction pattern of frog sciatic nerve in Ringer's solution. The pattern was taken using $30 \mathrm{kV}$ copper $\mathrm{K} \alpha$ nickel-filtered radiation, a specimen-to-film distance of $5.5 \mathrm{~cm}$, an exposure time of $\mathrm{I} h$ and the radial period was $d=17 \mathrm{I} \AA$. The first five orders of diffraction, $h=\mathbf{I}-5$, are visible. The first order $h=\mathrm{I}$ is better resolved on the original X-ray film as the reproduction magnifies the central X-ray beam scatter. Note the sharpness of the diffraction lines

Fig. 2. A point-focus low-angle X-ray diffraction pattern of frog sciatic nerve in Ringer's solution. The pattern was taken using $30 \mathrm{kV}$ copper $\mathrm{K} \alpha$ nıckel-filtered radiation, a specimen-to-film distance of $5.5 \mathrm{~cm}$, an exposure time of about I day and the radial period was $d=\mathrm{I} 7 \mathrm{I} \AA$. Diffraction orders $h=2,3,4,5$ are visible together with a higher order $h=1 \mathrm{I}$. The reflections are arced but the maximum intensity is on the equator. It should be noted that the first order, $h=\mathrm{I}$, was visible on the original X-ray film, but it is not resolved in the reproduction as it has been over-exposed in order to show the presence of the diffraction order $h=$ I I

patterns ${ }^{\mathbf{2 , 4}}$ taken with either pinhole or slit collimation do not easily lead to this conclusion. The interference function of the radial repeating unit is surprisingly sharp in view of the fact that the unit cell size is comparatively large and myelin consists of many different chemical components with a high water content.

When point focus is used, that is, both mirrors, the myelin reflections are seen to arc slightly with the origin of the patterns as the center of the arc. The peak intensity is on the equator in all cases. Fig. 2 shows a point-focus low-angle X-ray diffraction pattern from frog sciatic nerve in Ringer's solution. The exposure time was about $\mathrm{I}$ day. The first five diffraction orders of $d=$ I $\mathrm{I} \AA$ are visible on the original X-ray film, but in Fig. 2 the first order $h=\mathrm{I}$ is obscured by the central halo. Note the presence of the diffraction order $h=\mathrm{II}$. The arcing of the reflections will contribute to a certain amount of line broadening in line-focus patterns, and therefore the true interference function is even sharper than the one which would be derived from the consideration of Fig. I alone. 
Line-focus operation is normally used, and an exposure of $\mathrm{I}$ h suffices to show the first five diffraction orders. Longer exposure times of about Io-I $2 \mathrm{~h}$ using line operation showed additional orders. Peripheral nerve myelin specimens generally showed additional orders $h=6,8$ and II, while central nervous system myelin specimens showed additional orders $h=1,3,5,6$ and ro. Figs. 3, 4 and 5 show higher diffraction orders from peripheral nerve myeln and Fig. 6 shows higher diffraction orders from central nervous system myelin.
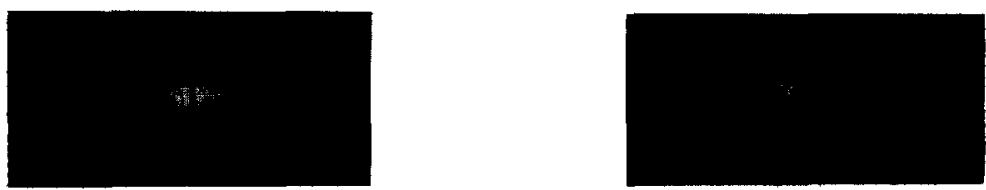

Fig. 3. A line-focus low-angle X-ray diffraction pattern of frog sciatic nerve in Ringer's solution. The pattern was taken using $30 \mathrm{kV}$ copper $\mathrm{K} \alpha$ nickel-filtered radiation, a specimen-to-film distance of $55 \mathrm{~cm}$, an exposure time of $\mathrm{I} 2 \mathrm{~h}$ and the radial period was $d=\mathrm{I} 7 \mathrm{I} \AA$ The central region is over-exposed in the reproduction in order to see the higher orders. Diffraction orders $h=2,3$, $4,5,6$ and II are visible.

Fig 4. A line-focus low-angle X-ray diffraction pattern of chicken sciatic nerve in Krebs-Ringer's solution. The pattern was taken using $30 \mathrm{kV}$ copper $\mathrm{K} \alpha$ nickel-filtered radiation, a specimen-to-film distance of $55 \mathrm{~cm}$, and exposure tıme of $15 \mathrm{~h}$ and the radial period was $d=182 \AA$. The central region is over-exposed in the reproduction obscuring orders $h=1,2,3,4$ which are, however, visible on the original X-ray film. Diffraction orders $h=5,6,8$, Io, I I and I 2 are shown.
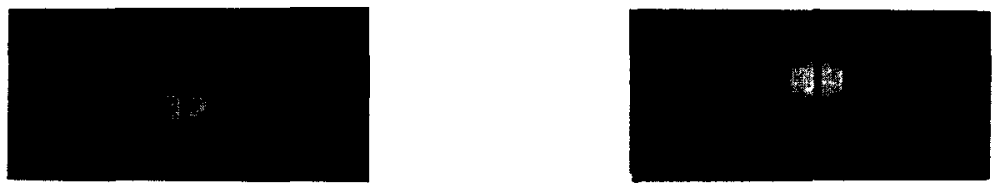

Fig. 5 A line-focus low-angle X-ray diffraction pattern of rat sciatic nerve in Krebs-Ringer's solution. The pattern was taken using $30 \mathrm{kV}$ copper $\mathrm{K} \alpha$ nickel-filtered radiation, a specimen-tofilm distance of $55 \mathrm{~cm}$, an exposure time of $\mathrm{I} 2 \mathrm{~h}$ and the radial period was $d=\mathrm{I} 76 \AA$. The central region is over-exposed in order to show the higher orders of diffraction Diffraction orders $h=$ $3,4,5,6,8$, and I I are clearly visible while weaker orders $h=7$, Io are just discernible

Fig. 6. A line-focus low-angle X-ray diffraction pattern of rat optic nerve in Krebs-Ringer's solution. The pattern was taken using $30 \mathrm{kV}$ copper $\mathrm{K} \alpha$ nickel-filtered radiation, a specimen-to-film distance of $55 \mathrm{~cm}$, an exposure time of $6 \mathrm{~h}$ and the radial period was $d=159 \AA$. The central region is over-exposed in order to show the higher orders of diffraction. Diffraction orders $h=4$ and Io are visible while the weaker order $h=6$ is just discernıble.

Fig. 3 shows a line-focus low-angle pattern of frog sciatic nerve in Ringer's solution. The exposure time was I $2 \mathrm{~h}$. The diffraction orders $h=\mathrm{I}-5$ are over-exposed, but the higher orders $h=6$ and $h=$ II are visible in Fig. 3 .

Fig. 4 shows a line-focus low-angle pattern of chicken sciatic nerve in KrebsRinger's solution. The exposure time was $\mathrm{I} 5 \mathrm{~h}$. The first five orders are overexposed, but orders $h=6,8$, I0, II, I2 of $d=$ I $82 \AA$ are visible in Fig. 4 .

Fig. 5 shows a line-focus low-angle pattern of rat sciatic nerve in Krebs-Ringer's solution. The exposure time was $\mathrm{I} 2 \mathrm{~h}$. The first five diffraction orders of $d=\mathrm{I} 76 \AA$ are over-exposed, but higher orders $h=6,8$, II are visible. Orders $h=7$, ro are weaker but can also be seen in Fig. 5 . 
Fig. 6 shows a line-focus low-angle pattern of rat optic nerve in Krebs-Ringer's solution. The exposure time was $6 \mathrm{~h}$. The first two strong orders $h=2$ and 4 of $d=$ I59 $\AA$ are over-exposed, but the higher order $h=$ Io is visible. Order $h=6$ is weaker but is also discernible in Fig. 6. Diffraction orders $h=\mathrm{I}, 3,5$ are seen on the original X-ray film.

Extra reflections are often seen in peripheral nerve myelin patterns (frog sciatic nerve) which are attributed to collagen. Collagen is known to be present in myelinated nerve. On the basis of spacing measurement and intensity comparison, three reflections are identified as diffraction orders $h=3,5$ and 9 of $d=670 \AA$ from a wet collagen ${ }^{10}$.

A regular feature of the low-angle pattern obtained from freshly dissected frog sciatic nerve in Ringer's solution was the presence of a rather faint diffuse band centered at about $d=25^{\circ} \AA$. A similar diffuse band obtained after storing frog sciatic nerve in Ringer's solution for several days has been previously reported ${ }^{11}$. In our case this reflection appears in frog sciatic nerve specimens stored in Ringer's solution for no longer than $\mathrm{I} h$ and with an exposure time as short as $3 \mathrm{~h}$, although $\mathrm{I} 2 \mathrm{~h}$ is normally required to register the diffuse pattern. The origin of this diffuse $25^{\circ} \AA$ is not known.

\section{Radial repeat distances}

Our measurements of the radial repeat distance from the various nerve specimens are reproducible. We note, however, that the actual magnitude of the period for any one particular variety of nerve does vary slightly depending on specimen preparation (composition of solution, time in solutions, temperature of solutions and temperature of specimen during the X-ray experiment). Our measurements of the period, $d$, for the various nerve specimens are in close agreement with previous work $^{2-4,12,13}$ when allowance is made for some differences in specimen preparation. Our values of $d$ are given in Table I.

In the case of frog and rat, we note that the radial repeat distance of the sciatic nerve exceeds that of the optic nerve by either I7 or I8 $\AA$, but the difference in the case of chicken is $27 \AA$.

\section{TABLE I}

VALUES OF $d$ OF PERIPHERAL NERVE MYELIN AND CENTRAL NERVOUS SYSTEM MYELIN FOR VARIOUS NERVE SPECIMENS

\begin{tabular}{|c|c|c|}
\hline \multirow[t]{2}{*}{ Nerve specimen } & \multicolumn{2}{|l|}{$d(A)$} \\
\hline & $\begin{array}{l}\text { Perpheral nerve } \\
\text { myelmn }\end{array}$ & Central nervous system myelin \\
\hline Frog sciatic nerve & I7I (cf. I7I, ref. 2) & - \\
\hline Rat sciatic nerve & $\mathrm{I}_{7} 6\left(c f . \mathrm{I}_{7} 8\right.$, ref. $\left.\mathrm{I} 2\right)$ & 一 \\
\hline Chicken sciatıc nerve & I $82\left(c f . \mathbf{I}_{3}\right.$, ref $\left.\mathrm{I}_{3}\right)$ & - \\
\hline Frog optic nerve & - & I54 (no comparison) \\
\hline Frog spinal cord & - & I53 (no comparison) \\
\hline Rat optic nerve & - & I 59 (cf. r6o, ref. 3$)$ \\
\hline Chicken optic nerve & - & I55 (cf. I6o (for pigeon optic nerve) ref. 4 ) \\
\hline Beef brain white matter & 一 & I57 (no comparison) \\
\hline
\end{tabular}




\section{Intensity variation}

If we confine our attention to the relative intensities, $I(h)$, of the first five orders of diffraction from peripheral nerve and central nervous system myelin, then a characteristic intensity variation between the diffraction orders is noted. There are two intensity variations: one for peripheral nerve myelin and one for central nervous system myelin. In order to establish this intensity variation, the intensities $I(h)$ on a relative scale for the first five diffraction orders for sciatıc and optic nerves from frog, rat and chicken are listed in Table II. For convenience, in each case the strongest intensity, $I(2)$, is given a value of unity. All three patterns of peripheral nerve myelin show a consistent intensity variation: $I(2)>I(4)>I(3)>I(5)>I(\mathrm{I})$. We note that relative intensity plots for frog and rat sciatıc nerves have been previously published $^{3}$, and these plots are in reasonable agreement with the values given in Table II. From Table II the intensity variation for the three central nervous system myelins is clearly different from that given by the three peripheral nerve myelins. All three patterns of central nervous system myelin show a consistent intensity variation: $I(2)>I(4)>I(\mathrm{I}) \approx I(3) \approx I(5)$ with $I(2)$ and $I(4)$ being much more intense. We note that our patterns of frog spinal cord and beef brain white matter both show the above characteristic central nervous system intensity varlation.

\section{Equatorial reflection at $15-16$ A}

The wide-angle pattern of fresh nerve ${ }^{1}$ shows a meridionally accentuated diffuse ring at $4.7 \AA$ (our wide-angle patterns are in the range of $4.5-4.7 \AA$ ) and an equatorial reflection at $\mathrm{I} 5-\mathrm{I} 6 \AA$. Our low-angle $\mathrm{X}$-ray camera examines the equatorial

TABLE II

RELATIVE VALUES OF INTENSITIES $I(h)$ FOR PERIPHERAL NERVE AND CENTRAL NERVOUS SYSTEM MYELIN

\begin{tabular}{|c|c|c|c|c|c|c|}
\hline \multirow[t]{2}{*}{ Nerve specimen } & \multirow[t]{2}{*}{$d(A)$} & \multicolumn{5}{|l|}{$h$} \\
\hline & & $I$ & 2 & 3 & 4 & 5 \\
\hline Frog sciatic nerve & $\mathbf{I} 7 \mathbf{I}$ & 0.013 & I.000 & o.I 69 & 0403 & 0084 \\
\hline Rat sciatic nerve & 176 & 0.018 & I.OOO & 0.204 & 0.563 & 0.118 \\
\hline Chıcken sciatıc nerve & 182 & 0.016 & I.OOO & 0.163 & 0514 & 0.067 \\
\hline Frog optic nerve & I 54 & 0.034 & 1.000 & 0.042 & 0.256 & 0.008 \\
\hline Rat optic nerve & I 59 & 0017 & 1.000 & 0.015 & 0224 & 0.023 \\
\hline Chicken optic nerve & 155 & O OI4 & 1.000 & 0.029 & o I 74 & 0005 \\
\hline
\end{tabular}

TABLE III

$d / h$ VALUES FOR VARIOUS NERVE SPECIMENS

\begin{tabular}{llll}
\hline Nerve specimen & $d(A)$ & $h$ & $d / h(A)$ \\
\hline Frog sciatic nerve & I7 & I I & I5 5 \\
Rat sciatic nerve & I76 & I I & I6. I \\
Chicken sciatic nerve & I82 & I I & I5 2, I6 5 \\
Frog optic nerve & I54 & Io & I5.4 \\
Rat optic nerve & I59 & Io & I5.9 \\
Chicken optic nerve & I55 & Io & I5.5 \\
& & & \\
\hline
\end{tabular}


reflections in fine detail and shows that the equatorial reflection at I5-I6 $\AA$ observed with a pinhole camera of moderate resolution consists of either one or more diffraction orders of the radial repeat.

The peripheral nerve myelin typically shows a strong IIth order, whereas central nervous system myelin shows the strong roth order. The $d / h$ values are given in Table III.

The spacing $d / h$ has a small variation of only $15.2-16.5 \AA$ for peripheral nerve and central nervous system myelin.

\section{Nerve myelin from fish}

Nerve myelin from fish has an intensity variation which is different from both the peripheral nerve and central nervous system myelin patterns. FINEAN ${ }^{4}$ has recorded three orders $(h=2,3,4)$ from lateral line, spinal cord and optic nerves of one variety of fish and has drawn attention to the comparatively weak $4^{\text {th }}$ order. HoGLUND AND RINGERTZ ${ }^{13}$ have recorded the same three orders from the lateral line of five varieties of fish and have also noted the weak $4^{\text {th }}$ order. Our low-angle patterns of lateral line, spinal cord and optic nerve from one variety of fish confirm and extend the earlier observations $\mathbf{4}^{\mathbf{4} 13}$. The intensity variation in our patterns is $I(2)>I(4)$ $\approx I(3)>I(\mathrm{I}) \approx I(5)$. Our measurements of the radial repeat distances are as follows: lateral line: $d=\mathrm{I} 59 \AA$, $c f$. I6o $\AA$, ref. 4 ; spinal cord, optic nerve $d={ }_{5} 6 \AA$, $c f$. I50 $\AA$, ref. 4 .

Additional diffraction orders $h=\mathrm{I}, 5,7,9$ and II are visible in our low-angle patterns from fish lateral line together with the orders $h=2,3,4$ previously reported ${ }^{4,13}$. The intensities $I(9)$ and $I(\mathbf{I I})$ are about equal. The $d / h$ values for $h=9, \mathbf{I I}$ are 17.7 and $14.5 \AA$. These values of $d / h$ differ from peripheral nerve and central nervous system myelin patterns. However, the average of these two $d / h$ values is about $\mathrm{r} 6 \AA$, which is the spacing of the equatorial reflection seen in wide-angle pattern (taken with moderate resolution).

An additional feature of fish myelin low-angle patterns is the observation of a second radial repeat period. The periods quoted above refer to the most intense pattern. The second period $d=\mathrm{I} 84 \AA$ ( $2 \%$ accuracy) is found in lateral line, $d=$ I83 $\AA$ in fish optic nerve and $d=I 82 \AA$ in fish spinal cord. The intensity variation of this larger radial repeat distance is similar to that of peripheral nerve myelin.

\section{DISCUSSION}

The observation of very sharp diffraction orders in the low-angle X-ray diffraction pattern of nerve myelin shows that the ordering of the concentric layers of myelin is remarkably precise. This is somewhat surprising in view of the fairly large center-to-center distance between layers, the complex composition of the layers and the high water content. The low-angle pattern of peripheral nerve myelin consisting of the first five diffraction orders is well-known; these orders have comparatively strong intensities. However, in this study higher diffraction orders $(h>5)$ have been recorded. These diffraction orders, $h>5$, only have small intensities, partly because of the cylindrical layering involved and partly because of the kind of membrane structure present.

The low-angle $\mathrm{X}$-ray diffraction patterns of peripheral nerve and central nervous 
system myelin show essential differences in radial repeat distances and in the intensity variation of the diffraction orders. The radial repeat distances for peripheral nerve myelin are I7I-I82 $\AA$, while those for central nervous system myelin are I53- I59 $\AA$. The radial periods for peripheral nerve myelin are consistently larger than for central nervous system myelın by $17-27 \AA$ for the various sources studied. Both kinds of myelin show strong diffraction in the region of $\mathrm{I}^{-1} \mathrm{I} 6 \AA$ and this suggests that the same structural element responsible for this diffraction is present in both peripheral nerve and central nervous system myelin.

Peripheral nerve and central nervous system myelın show different intensity variations which are independent of the source in our three cases (frog, rat and chicken). This implies that peripheral nerve myelin from these sources all have a similar structure, and the same conjecture applies to central nervous system myelin. However, this implication is likely to be only a first approximation because it can be traced to an intensity variation which includes only the first five orders of diffraction. Differences in intensity variation will no doubt show up in myelins from different sources either when accurate intensities are considered, or else when higher orders of diffraction are taken into account.

We note that myelin from fish shows two low-angle X-ray diffraction patterns; the stronger pattern has the smaller radial repeat distance somewhat similar to central nervous system myelin, but the intensity variation is significantly different from central nervous system myelin. The case of fish is anomalous and merits further study.

An interesting feature of the present study is the observation of higher orders of diffraction in both the peripheral nerve and central nervous system myelin patterns. Highers diffraction orders out to a minimum spacing of $15^{-16} \AA$ have been recorded. If these orders can be used in a Fourier analysis, then the resolution $(\Delta r)$ of the Fourier map is $\Delta r \approx 7.5-8 \AA$. This then represents a persistent challenge to find more detailed models for the structure of nerve.

\section{ACKNOWLEDGEMENT}

This work was supported by United States Public Health Service grant GM 09796.

\section{REFERENCES}

I F. O. Schmitt, R. S. Bear and G. L. Clark, Radiology, 25 (I935) I31.

2 F. O. Schmitt, R. S. Bear and K. J. Palmer, J. Cellular Comp. Physiol., i8 (I94I) 3 I.

3 J. B. Finean, Koninkl. Vlaam. Acad. Wetenschap. Letter. Schone Kunsten Belg., Kl. Wetenschap., Ist Intern. Colloq. Biochem. Probl. Lipiden, Brussels, 1953, I953, p. 82.

4 J. B. Finean, in J. N. Cummings, Modern Scientific Aspects of Neurology, Edward Arnold, London, 1960.

5 A. E. BlaUrock and C. R. Worthington, Brophys. J., 6 (I966) 305.

6 G F Elliott and C R. Worthington, J. Ultrastruct. Res., 9 (I963) I66.

7 C R. Worthington and A. E. BlaUrock, Nature, 218 (1968) 87.

$8 \mathrm{~J}$. K Blasie, M M. Dewey, A. E. Blaurock and C. R. Worthington, J. Mol. Biol, i 4 (1965) 143 .

9 B M. Millman, to be published.

io S. G. Tominn and C. R Worthington, Proc. Roy. Soc. London, Ser. A, 235 (1956) I89.

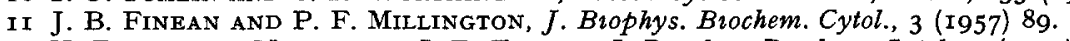

12 H. Fernandez-Moran and J. B. Finean, J. Brophys. Brochem. Cytol., 3 (1957) 725.

I 3 G. Hoglund and H. Ringertz, Acta. Physiol. Scand., 5 I (I960) 290. 